\title{
EL MÉTODO DELPHI. SU IMPLEMENTACIÓN EN UNA ESTRATEGIA DIDÁCTICA PARA LA ENSEÑANZA DE LAS DEMOSTRACIONES GEOMÉTRICAS
}

\begin{abstract}
María de Lourdes Bravo Estévez, Universidad de Cienfuegos. Cuba. José Joaquín Arrieta Gallastegui, Universidad de Oviedo. España.
\end{abstract}

Como parte del Programa de Doctorado "Diseño y Desarrollo del Curriculum" de las universidades de Cienfuegos (Cuba) y Oviedo (España) realizamos la investigación "Una estrategia didáctica para la enseñanza de las demostraciones geométricas", fundamentada en la necesidad de buscar nuevos procedimientos que contribuyeran a la elevación del proceso de enseñanza-aprendizaje de la disciplina Geometría en el nivel superior, ante la problemática de ¿cómo desarrollar la habilidad "demostrar" para resolver los problemas geométricos de demostración durante el tratamiento de la Estereometría en los estudiantes en formación para profesores de Matemáticas?.

En la investigación perseguimos como objetivo la elaboración e implementación de una estrategia didáctica para la enseñanza de las demostraciones geométricas centrada en un sistema de acciones para el desarrollo de la habilidad "demostrar", que permita a los estudiantes en formación para profesores de Matemáticas resolver con mayor éxito los problemas geométricos de la Estereometría.

El estudio de campo de la investigación lo desarrollamos en tres etapas: la del criterio de expertos, la fase experimental y la encuesta a los estudiantes, siguiendo una estrategia de triangulación. Este trabajo se refiere a la primera de estas etapas, trabajo en que describimos la planificación, desarrollo y discusión de los resultados obtenidos con la puesta en práctica del Método Delphi.

Ruiz e Ispizua (1989) describen la técnica Delphi como un método de investigación sociológica, que independientemente de que pertenece al tipo de entrevista de profundidad en grupo, se aparta de ellas agregando características particulares. Es una técnica grupal de análisis de opinión, parte de un supuesto fundamental y de que el criterio de un individuo particular es menos fiable que el de un grupo de personas en igualdad de condiciones, en general utiliza e investiga la opinión de expertos.

Varios son los autores que han aportado una definición de este método, aunque para Konow y Pérez (1990) intentar dar una definición es como limitar el alcance y contenido del método, por lo que es más aconsejable dar una descripción general de sus características, limitaciones, usos y aplicaciones. Por nuestros intereses nos parece adecuado, para su comprensión en la aplicación del análisis de datos, realizar una descripción de sus elementos fundamentales.

Parisca (1995) considera que el Método Delphi se basa en el principio de la inteligencia colectiva y que trata de lograr un consenso de opiniones expresadas individualmente por un grupo de personas seleccionadas cuidadosamente como expertos calificados en torno al tema, por medio de la iteración sucesiva de un cuestionario retroalimentado de los resultados promedio de la ronda anterior, aplicando cálculos estadísticos. 
En nuestro caso es utilizado el Método Delphi para evaluar las características de la estrategia didáctica y en particular del sistema de acciones para el desarrollo de la habilidad "demostrar" por expertos en distintas áreas del conocimiento matemático, es aplicado como una previsión del comportamiento de las características de la estrategia y del sistema a estudiar, que ya son conocidos por lo que se indaga sobre su posible proceder.

Las principales características del método están dadas por el anonimato de los participantes (excepto el investigador), iteración (manejar tantas rondas como sean necesarias), retroalimentación (feedback) controlada, sin presiones para la conformidad, respuesta de grupo en forma estadística (el grado de consenso se procesa por medio de técnicas estadísticas) y justificación de respuestas (discrepancias/consenso).

Suelen distinguirse tres etapas o fases fundamentales en la aplicación del método, según la variada bibliografía consultada al respecto, que son:

1. Fase preliminar. Se delimita el contexto, los objetivos, el diseño, los elementos básicos del trabajo y la selección de los expertos.

2. Fase exploratoria. Elaboración y aplicación de los cuestionarios según sucesivas vueltas, de tal forma que con las respuestas más comunes de la primera se confecciona la siguiente.

3. Fase final. Análisis estadísticos y presentación de la información.

Fases que representamos en el siguiente esquema entrelazado:

ESQUEMA DE DESARROLLO DEL MÉTODO DELPHI

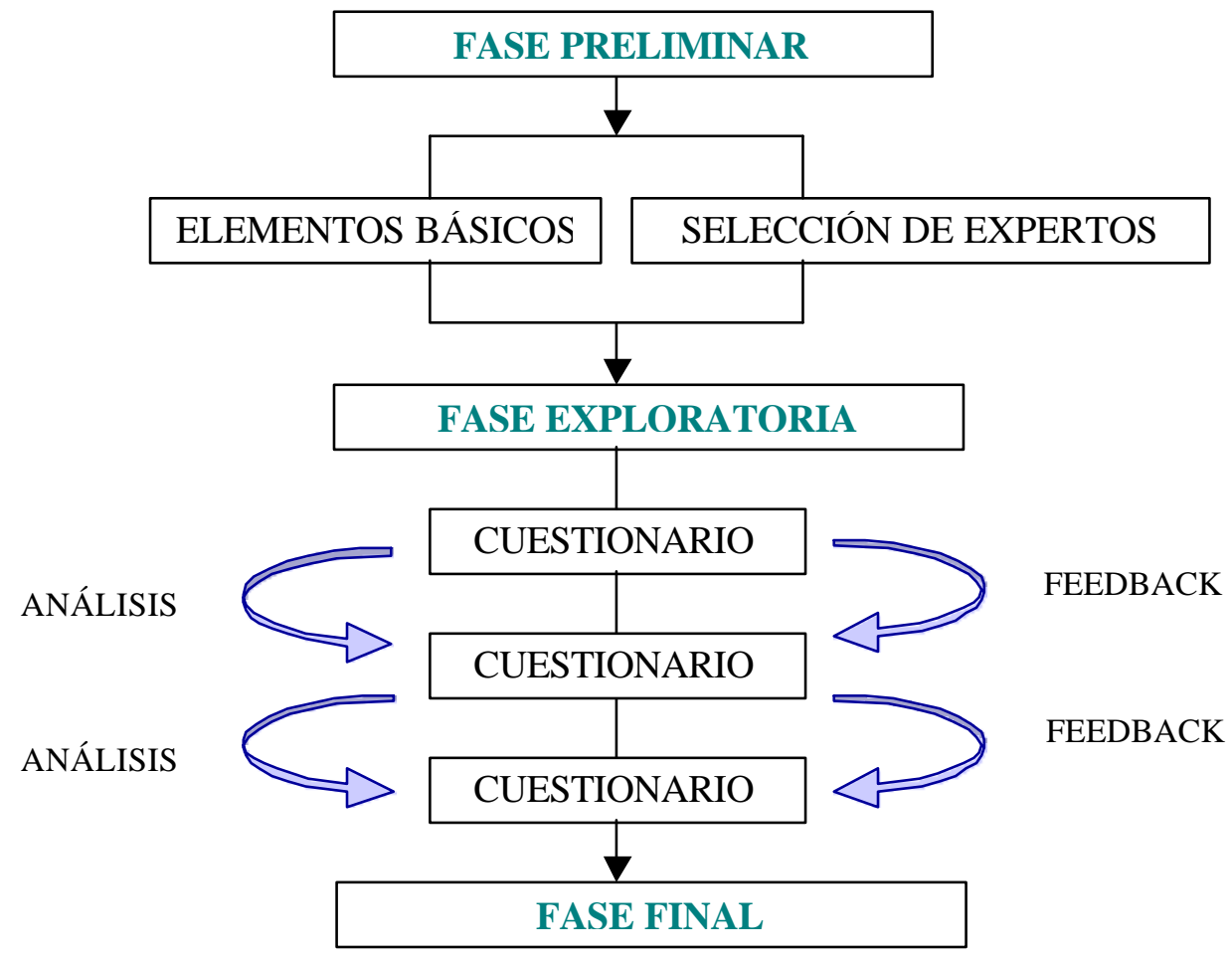

Para la aplicación del método es necesario considerar metodológicamente dos aspectos básicos de su caracterización sobre los cuales se sustenta, que son: 
- La selección del grupo de expertos a encuestar: personas conocedoras, con reconocida competencia y con experiencia en el tema que garantice la confiabilidad de los resultados, creativos e interesados en participar.

Por la limitación de tiempo y recursos determinamos que el número de expertos participante en la investigación que desarrollamos no fuera muy numeroso, aunque se corresponde con el intervalo sugerido por los autores Ruiz e Ispizua (1989).

- Elaboración de los cuestionarios: tener en cuenta la teoría de la comunicación, con mecanismos que reduzcan los sesgos en las respuestas, preguntas claras, precisas e independientes. Suelen ser preguntas cuantitativas para calcular medias y rangos, y cualitativas para la justificación de sus opiniones.

Konow y Pérez (1990) distinguen distintos tipos de preguntas para la elaboración de los cuestionarios. Nosotros utilizamos las preguntas abiertas para señalar justificaciones de una determinada estimación y mencionar factores que pueden influir en el comportamiento de una determinada cuestión, de las preguntas cerradas escogimos las de votación para votar por una o más de las alternativas presentadas y, las preguntas de ranking para ordenar siguiendo un determinado criterio una serie de características en forma desordenada. En cierta forma también empleamos las preguntas de control haciendo dos veces la misma pregunta de forma distinta o indirecta.

La mayoría de los autores consultados que han estudiado el método opina que se realicen más de dos vueltas y que a partir de la cuarta ya se mantienen los mismos resultados, por lo que nosotros entendimos realizar tres cuestionarios.

Cada respuesta fue ponderada de acuerdo al nivel de confianza particular de cada respuesta y se definió el consenso según el tipo de pregunta, para nuestro caso estuvo determinado en las preguntas de votación en las que podían elegir más de una alternativa al menos el $60 \%$ del total de alternativas ponderadas y en el caso de que sólo fuera una respuesta, el 40\%. En la pregunta de ranking la suma del valor asignado (Sj) consideramos que estuviera por debajo de la media $(\bar{S})$ del rango de valores (donde interviene el número de cuestiones generales y número de expertos). Para el indicador de concordancia de los criterios de expertos empleamos técnicas de diseño experimental no paramétrica.

Los autores consultados opinan como ventajas del método, que permite obtener información de puntos de vista sobre temas muy amplios y específicos, que el horizonte de análisis puede ser variado y que permite la participación de un gran número de personas sin dificultades con anonimato y retroalimentación. Plantean como desventajas del método, que no es muy fiable porque incluso los más eminentes especialistas pueden equivocarse, así como su tiempo de ejecución y la masiva participación.

Para la aplicación del método a partir de lo expresado anteriormente tuvimos en cuenta los siguientes pasos lógicos:

\section{Planificación del criterio de expertos}

- Concepción inicial del problema.

- Selección de los expertos.

- Los componentes o elementos necesarios para llevar a cabo el trabajo. 
2. Elaboración y aplicación de las encuestas.

3. Procesamiento y análisis de información.

Estos pasos son descritos a continuación.

En la planificación del criterio de expertos partimos de la concepción inicial del problema y la selección de los expertos como pasos previos fundamentales para la aplicación del criterio. En lo referido a la concepción inicial definimos los elementos básicos del trabajo que son los siguientes:

- El objetivo a alcanzar.

Basado en conocer la opinión de un grupo de expertos sobre las características de la estrategia didáctica propuesta, como un elemento que influirá en la validación del experimento para dilucidar la efectividad de la estrategia en el desarrollo de la habilidad "demostrar" desde otro ángulo, el de los expertos.

- La situación actual.

Dada por las insuficiencias en el desarrollo de la habilidad "demostrar" que presentan los estudiantes en formación para profesores de Matemáticas en el estudio de la Estereometría. Situación que queda fundamentada por la aplicación de instrumentos pedagógicos como: encuestas, entrevistas, observaciones de clases, así como por el análisis del rendimiento académico de los estudiantes en la asignatura "Estereometría" y la propia experiencia profesional.

- Los componentes o elementos necesarios para llevar a cabo el trabajo.

Son los componentes que conforman la estrategia didáctica para la enseñanza de las demostraciones geométricas propuesta en este trabajo, en especial del sistema de acciones para el desarrollo de la habilidad "demostrar" acompañado por un conjunto de consideraciones didácticas.

Los expertos se seleccionaron según las características siguientes: años de experiencia como profesor o profesora (en especial los dedicados a la formación del profesorado y en particular los referidos a las disciplinas de Geometría y/o Metodología de la Enseñanza de la Matemática), idoneidad en el sector educacional, prestigio entre el colectivo de profesores y estudiantes, creatividad al impartir sus clases, disposición para participar en la validación, capacidad de análisis y pensamiento lógico, espíritu colectivista y autocrítico.

Elegir los expertos atendiendo a las características mencionadas propicia obtener resultados con calidad, junto a otras cualidades propias de éstos como pueden ser: la seriedad, la honestidad, la sinceridad, la responsabilidad y otras en este sentido, que hacen que las opiniones brindadas sean confiables y válidas para el objetivo propuesto.

En nuestro caso seleccionamos diez expertos con el título de Licenciado en Educación Especialidad Matemática; dos de ellos con el grado científico de Doctor en Ciencias Pedagógicas y Ciencias Matemáticas, respectivamente. Desglosados por disciplinas de la siguiente forma: cuatro expertos pertenecientes a Geometría, tres a Metodología de la Enseñanza de la Matemática y el resto a Análisis Matemático, Álgebra y Metodología de la Investigación Pedagógica.

Todos los expertos seleccionados tienen más de veinte años de experiencia en educación y han transitado por los distintos niveles de enseñanza, además de ocupar cargos de dirección en la ens eñanza 
de las Matemáticas. Es significativo destacar que el promedio de años de experiencia en la Educación Superior, particularmente los dedicados a la formación de profesores de Matemáticas, es de nueve años. Sus evaluaciones profesionales están en el rango entre bien y excelente, contando con suficiente prestigio entre el colectivo de estudiantes y trabajadores de la institución.

Una vez conformado el paso anterior se procede a la elaboración y aplicación de las encuestas según cada ronda de preguntas, el procedimiento seguido se representa mediante el siguiente cuadro:

\begin{tabular}{|l|lr|l|}
\hline \multicolumn{1}{|c|}{ OBJETIVOS } & \multicolumn{1}{|c|}{ ENCUESTAS } & \multicolumn{1}{|c|}{ PREGUNTAS } \\
\hline $\begin{array}{l}\text { Búsqueda de elementos comunes y esenciales en las } \\
\text { valoraciones de los expertos. }\end{array}$ & $\begin{array}{l}\text { Primera ronda de } \\
\text { preguntas }\end{array}$ & Abiertas \\
\hline $\begin{array}{l}\text { Búsqueda de cuestiones generales más comunes en } \\
\text { cada pregunta. }\end{array}$ & $\begin{array}{l}\text { Segunda ronda de } \\
\text { preguntas }\end{array}$ & Cerradas \\
\hline Búsqueda de concordancia entre los expertos & $\begin{array}{l}\text { Tercera ronda de } \\
\text { preguntas }\end{array}$ & Cerradas \\
\hline
\end{tabular}

Para el procesamiento y análisis de la información se tiene en cuenta el tipo de pregunta si es abierta o cerrada y se valora desde lo cualitativo o cuantitativo respectivamente.

El análisis cualitativo es fundamentalmente para la primera ronda de preguntas que es de tipo abierta, se leen detalladamente cada una de las respuestas y se resumen los elementos más comunes y esenciales para elaborar la ronda siguiente. Lo cuantitativo en general es para la segunda y tercera ronda de preguntas, que son de tipo cerrada.

En la segunda ronda las características cuantitativas están asociadas a atributos, donde solamente será posible asignar dos valores a las variables. Cuando se presenta la característica deseada, se le asigna el valor 1 y si ésta no se presenta se asigna el valor 0 . Los expertos pueden seleccionar más de una característica en cada pregunta, por lo que luego de sumar para cada característica los valores deseados, se tomará como resultado aquellas que representen el $60 \%$ o más.

La primera parte de la última pregunta es distinta del resto, sólo pueden brindar una característica de las tres posibilidades, según una escala relacionada con el nivel de importancia otorgado a la estrategia didáctica, por lo que escogemos aquella que represente como mínimo el $40 \%$.

En la última ronda, el procesamiento de la información es de carácter cuantitativo, tipo de información que puede procesarse a partir de técnicas de diseño experimental no paramétrico (utilizamos básicamente los datos estadísticos propuestos por Oñate, Ramos y Díaz, 1990).

Se le asigna un rango a cada evaluación dada por el experto a las preguntas de la tercera ronda, de la siguiente manera:

- Valor 1

Evaluación mayor.

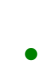

- $\mathrm{n}$ Evaluación menor.

La suma de rangos que se obtiene a partir de los valores ya definidos para cada pregunta se denota por $S_{\mathrm{j}}$ y se representa por

$S_{j}=\sum_{i=1}^{m} R_{i j}$ ( $\mathrm{R}_{\mathrm{ij}}$ el rango asociado a la evaluación del experto “i” a la pregunta "j"). 
Si el experto empleara la misma evaluación para más de una pregunta, el rango será igual a la media aritmética de las posiciones que deben ser adjudicadas.

El valor de $S_{j}$ es utilizado para comparar la importancia de diferentes respuestas, de modo que un menor valor significará una mayor importancia. Además se empleará para buscar el coeficiente de concordancia.

Se define también la media de la suma de rangos de cada pregunta "j" denotada por $\bar{S}$, la que se calcula según la fórmula:

$$
\bar{S}=\frac{\sum_{j=1}^{n} S_{j}}{n}=\frac{m(n+1)}{2}
$$

Con los valores de $S_{j}$ y $\bar{S}$ es posible calcular el coeficiente de concordancia Kendall (denotado por k) cuando no existen ligaduras como:

$$
\mathrm{k}=\frac{12 \sum_{j=1}^{n}\left(S_{j}-\bar{S}\right)^{2}}{m^{2}\left(n^{3}-n\right)-m \sum_{i=1}^{m} T_{i}}=\frac{12 \sum_{j=1}^{n} S_{j}^{2}-n \bar{S}^{2}}{m^{2}\left(n^{3}-n\right)-m \sum_{i=1}^{m} T_{i}}
$$

En la fórmula anterior $T_{i}$ representa el resultado de los rangos iguales (Ilamados también ligas), que ofreció el experto "i" para las preguntas, que se calcula como sigue:

$$
T_{i}=\frac{\sum_{i=1}^{l}\left(t^{3}-t\right)}{12} \text {, donde I y } \mathrm{t} \text { representan los siguientes aspectos: }
$$

I: número de grupos con rangos iguales para el experto "i".

t: número de observaciones dentro de cada uno de los grupos para el experto "i".

Los valores del coeficiente " $k$ " deben oscilar entre 0 y $1(0<k<1)$, si $k$ alcanza el valor uno $(k=1)$ entonces existe una concordancia total de criterios, mientras mayor sea el valor de $k$, es decir, cuanto más se acerque a uno, mayor será la concordancia entre los expertos.

Luego se aplica la Prueba de Significación de Hipótesis, planteándose la hipótesis nula y la alternativa de la siguiente forma:

$\mathrm{H}_{0}$ : no existe comunidad de preferencia entre los expertos, $\mathrm{k}=0$

$\mathrm{H}_{1}$ : existe comunidad de preferencia entre los expertos, $\mathrm{k} \neq 0$

Se determina Chi-cuadrado calculado como: $X_{c a l}^{2}=m(n-1) k$

Por otra parte, se busca el Chi-cuadrado tabulado en la tabla del percentil de la distribución Chicuadrado con un nivel de significación $\quad$ yn- 1 grados de libertad, representado por $X_{t a b}^{2}=\mathrm{X}_{\alpha ; n-1}^{2}$ 
Se compara $X_{c a l}^{2}$ y $X_{t a b}^{2}$, si se obtiene que $X_{c a l}^{2}>X_{t a b}^{2}$ entonces se rechaza $\mathrm{H}_{0}$ y se infiere que existe concordancia de criterios preferenciales entre los expertos al considerar válida la hipótesis alternativa $\mathrm{H}_{1}$.

Por último, resumimos básicamente en qué aspectos están de acuerdo los expertos como resultado del procesamiento y análisis de los criterios ofrecidos por ellos.

Después de aplicada la primera ronda de pregunta a los expertos se obtienen los siguientes resultados como aspectos más comunes y esenciales:

- La estrategia didáctica posee características novedosas que contribuyen al desarrollo de la habilidad "demostrar" en el estudio de la Geometría por:

- El diseño y ejecución del Curso Facultativo sobre modelos geométricos en que se potencia el desarrollo de la imaginación espacial.

- Las actividades de carácter metodológico encaminadas a la ejecución del sistema de acciones para el desarrollo de la habilidad "demostrar" junto a sus consideraciones didácticas.

- La proyección de un trabajo integral de la habilidad "demostrar" desde lo académico, laboral e investigativo.

- Sobre el sistema de acciones para el desarrollo de la habilidad "demostrar" en Geometría puede valorarse que:

- El sistema por sus acciones contribuye al desarrollo de la imaginación espacial.

- Las funciones de orientación, ejecución y control permiten un trabajo secuencial y graduado en la aplicación del sistema.

- Es un sistema generalizador, por lo que es aplicable a otros campos de la ciencia.

- Las acciones para resolver un problema geométrico de demostración se estructuran como un sistema.

- Los requisitos que cumplen las acciones del sistema hacen de este un sistema en sí.

- La aplicación de la estrategia didáctica es importante para el desarrollo de la habilidad "demostrar" porque:

- Puede aplicarse en otros campos de las Matemáticas o fuera de ésta.

- La estrategia contribuye a fomentar el valor formativo de las demostraciones geométricas.

- Potencia el desarrollo de forma gradual de cada una de las acciones que conforman la habilidad.

De la segunda ronda de preguntas se obtiene las siguientes conclusiones por preguntas:

Pregunta 1: 
(A) La estrategia didáctica posee características novedosas que contribuyen al desarrollo de la habilidad "demostrar" en el estudio de la Estereometría por la proyección de un trabajo integral de la habilidad "demostrar" desde lo académico, laboral e investigativo. (80\%)

\section{Pregunta 2:}

(B) Las acciones de la habilidad para resolver un problema geométrico de demostración se estructuran como un sistema. (100\%).

(C) El sistema por sus acciones contribuye al desarrollo de la imaginación espacial. (60\%).

(D) Es un sistema generalizador, por lo que es aplicable a otros campos de la ciencia. (60\%).

Pregunta 3 (Primera parte):

(E) La aplicación de la estrategia didáctica es muy importante para el desarrollo de la habilidad "demostrar". (90\%)

Pregunta 3 (Segunda Parte):

(F) La estrategia didáctica es importante porque contribuye a fomentar el valor formativo de las demostraciones geométricas. (80\%).

(G) La estrategia didáctica es importante porque potencia el desarrollo de forma gradual de cada una de las acciones que conforman la habilidad. (70\%).

Las conclusiones parciales obtenidas, como elementos más comunes, en cada una de las preguntas realizadas constituyen el cuerpo teórico de cuestiones generales para conformar la ronda siguiente, es decir, el tercer cuestionario (para buscar concordancia entre los expertos) queda conformado por las siete cuestiones generales descritas.

Los expertos deben asignarle un rango a las cuestiones generales recogidas en los incisos "j", según una escala ascendente del 1 al 7 , comenzando por el 1 como la evaluación mayor hasta llegar al 7 como la evaluación menor, obteniéndose la suma de rangos S para cada inciso. La comparación de los valores obtenidos para $S_{j}$ conduce a que los menores resultados corresponden a los incisos (B), (A) y (C) tomados en orden ascendente, con diferencias significativas de los dos primeros con respecto al tercero.

Calculamos la media $\bar{S}$ de la suma de rangos de cada inciso "j" por la fórmula (1) y obtenemos para $\mathrm{m}=10$ (números de expertos) y $\mathrm{n}=7$ (número de cuestiones):

$$
\bar{S}=\frac{10(7+1)}{2}=40
$$

Con el valor obtenido de $S$ y $\bar{S}$, y los cálculos parciales auxiliares que siguen, determinamos el coeficiente "k" de Kendall cuando no existen ligaduras por la fórmula (2) de la siguiente forma:

$$
\sum_{j=1}^{7} S_{j}^{2}-7 \bar{S}^{2}=(361+289+1369+2025+3249+2916+2601)-7(40)=1610
$$

Como todas las evaluaciones realizadas por los expertos "i" son diferentes, entonces el valor de $T_{i}$ $=0$. 
El coeficiente $\mathrm{k}$ resulta:

$$
\mathrm{k}=\frac{12(1610)}{10^{2}\left(7^{3}-7\right)}=\frac{19320}{100(336)}=\frac{19320}{33600}=0,575
$$

Obtenemos que $0<\mathrm{k}<1$, aunque este no es muy alto, es aceptable, por lo que están básicamente de acuerdo los expertos.

Para la Prueba de Significación de Hipótesis se calcula $X_{c a l}^{2}$ por la fórmula (3) como sigue: $X_{c a l}^{2}=$ 10. $6(0,575)=34,5$.

Buscamos en la tabla del percentil de la distribución Chi-cuadrado con un nivel de significación $\alpha=$ 0,05 y 6 grados de libertad el $X_{\text {tab }}^{2}$ obteniendo: $X_{0,05 ; 6}^{2}=12,592$.

Como 34,5 > 12,592 entonces se rechaza la hipótesis nula $\left(H_{0}\right)$ de que no existe comunidad de preferencia entre los expertos y se considera válida la hipótesis alternativa $\left(H_{1}\right)$ de que existe comunidad de preferencia entre los expertos.

Los resultados obtenidos en el criterio de expertos nos permiten arribar a las siguientes conclusiones:

- Con la aplicación del Método Delphi, resulta que existe concordancia de criterios preferenciales entre los expertos sobre las características de la estrategia didáctica que contribuyen al desarrollo de la habilidad "demostrar".

- En la validación del criterio de expertos se destacan como características preferencias de la estrategia para la enseñanza de las demostraciones geométricas las siguientes:

- Las acciones de la habilidad para resolver un problema geométrico de demostración se estructuran como un sistema.

- La estrategia didáctica posee características novedosas que contribuyen al desarrollo de la habilidad "demostrar" en el estudio de la Geometría por la proyección de un trabajo integral de la habilidad "demostrar" desde lo académico, laboral e investigativo.

- El sistema por sus acciones contribuye al desarrollo de la imaginación espacial.

\section{BIBLIOGRAFÍA}

Bravo EstéVEZ, María L. (2000). Propuesta de un sistema de acciones didácticas para la enseñanza de las demostraciones en Estereometría. Trabajo de investigación, Universidad de Oviedo. (España).

Bravo EstéVEZ, María L. (2002). Una propuesta didáctica para el desarrollo de la habilidad "demostrar" en el estudio de la Estereometría. Tesis de maestría, Universidad de Cienfuegos. (Cuba).

BRAVo EstÉVEZ, María L. (2002). Una estrategia didáctica para la enseñanza de las demostraciones geométricas. Tesis doctoral, Universidad de Oviedo. (España). 
GUTIÉRREZ ROdRíGUEZ, Ángel (1991). La investigación en Didáctica de las Matemáticas. En Gutiérrez Rodríguez, A. (Ed.). Área de conocimiento. Didáctica de la Matemática. Madrid: Síntesis, S.A, 149191.

KILPATRIC, J. (1996). Valoración de la investigación en Didáctica de las Matemáticas: más allá del valor aparente. En Puig, L. y Calderón, J. Investigación y Didáctica de las Matemáticas. Madrid: Ministerio de Educación y Ciencia, 31-48.

KONOW, I. y PÉREZ, G. (1990). Método Delphi. En: http://geocities.com/Pentagon/Quarters/7578/pros0103.html

oñate martínez, N., Ramos morales, L y Díaz Armesto, A. [1990]. Utilización del Método Delphi en la pronosticación: una experiencia inicial. La Habana: Instituto de Investigaciones Económicas de la Junta Central de Planificación.

PARISCA, S. (1995). El método Delphi. Gestión tecnológica y competitividad. En Parisca, S. Estrategia y filosofía para alcanzar la calidad total y el éxito en la gestión impresional. La Habana: Academia, 129-130.

PÉREZ ANDRÉS, C. (2002). ¿Deben estar las técnicas de consenso incluidas entre las técnicas de investigación cualitativa?. En: http://www.msc.es/salud/epidemiologia/resp/200004/editorial1.htm

Ruiz OlabuÉnAga, J. e ISPIZUA, M. A. (1989). La técnica Delphi. En Ruiz Olabuénaga, J. e Ispizua, M. A. La descodificación de la vida cotidiana. Métodos de investigación cualitativa. Bilbao, 171-179. 


\title{
Contactar
}

Revista lberoamericana de Educación

\author{
Principal OEI
}

